\title{
ON DISCRETE CHAMBER-TRANSITIVE AUTOMORPHISM GROUPS OF AFFINE BUILDINGS
}

\author{
W. M. KANTOR, R. A. LIEBLER AND J. TITS
}

1. Introduction. Let $\Delta$ be the affine building of a simple adjoint algebraic group $\mathcal{G}$ of relative rank $\geq 2$ over a locally compact local field $K$. Let Aut $\Delta$ (resp. $\Sigma$ Aut $\Delta$ ) denote the group of type-preserving (resp. of all) automorphisms of $\Delta$. Note that $\Sigma$ Aut $\Delta$ contains the group $\mathcal{G}(K)$ of $K$-rational points of $\mathcal{G}$. We will be interested in discrete subgroups of Aut $\Delta$ which are chamber-transitive on $\Delta$. It is extremely rare that such groups exist and, as can therefore be expected, exceptions are interesting phenomena; our purpose is to list them all (see the theorem below). In order to describe them we must first introduce some notation.

Let $f$ be a quadratic form in $n$ variables over $\mathbf{Q}_{p}$ with coefficients in $\mathbf{Z}$. We let $\operatorname{P} \Omega(f, \mathbf{Z}[1 / p])$ denote the intersection $\operatorname{PSO}\left(f, \mathbf{Q}_{p}\right)^{\prime} \cap \operatorname{PGL}(n, \mathbf{Z}[1 / p])$ within $\operatorname{PGL}\left(n, \mathbf{Q}_{p}\right)$, and similarly $\operatorname{PGO}(f, \mathbf{Z}[1 / p])=\operatorname{PGO}\left(f, \mathbf{Q}_{p}\right) \cap \operatorname{PGL}(n, \mathbf{Z}[1 / p])$. In the following list, $\Gamma$ will always be a chamber-transitive subgroup of Aut $\Delta$. The fundamental quadratic form (over $\mathbf{Z}$ ) of the root lattice of type $A_{n}, B_{n}$, $E_{n}$, normalized so that the long roots have squared length 2 , will be denoted by $a_{n}, b_{n}, e_{n}$, respectively; note that $b_{n}$ is $\sum_{1}^{n} x_{i}^{2}$.

(i) Let $f=e_{8}, b_{7}, a_{6}, b_{6}, e_{6}$, or $a_{5}$, and let $\Delta$ be the affine building of $\operatorname{PSO}\left(f, \mathbf{Q}_{2}\right)$. Here $\Gamma$ can be any group between $\Gamma_{\min }=\operatorname{P} \Omega(f, \mathbf{Z}[1 / 2])$ and $\Gamma_{\max }=\operatorname{PGO}(f, \mathbf{Z}[1 / 2]) \cap$ Aut $\Delta$. The quotient $\Gamma_{\max } / \Gamma_{\min }$ is elementary abelian of order $1,1,1,4,2$, or 2 , respectively, and $\Gamma_{\max }$ is generated by $\Gamma_{\min }$ and reflections.

(ii) Let $f=b_{5}, e_{6}$, or $b_{6}^{\prime}=\sum_{1}^{5} x_{i}^{2}+3 x_{6}^{2}$, and let $\Delta$ be the building of $\operatorname{PSO}\left(f, \mathbf{Q}_{3}\right)$. The group $\Gamma_{\max }(f)=\operatorname{PGO}(f, \mathbf{Z}[1 / 3]) \cap$ Aut $\Delta$ has 3,5 , or 9 conjugacy classes of chamber-transitive subgroups $\Gamma$. Passage $\bmod 2 \operatorname{maps}$ $\Gamma_{\max }\left(b_{5}\right)$ onto the symmetric group $S_{5}$, and the preimages in $\Gamma_{\max }\left(b_{5}\right)$ of $S_{5}$, $A_{5}$, or a group of order 20 form the 3 desired conjugacy classes of groups $\Gamma$. The forms $e_{6}$ and $b_{6}^{\prime}$ are rationally equivalent, and hence the buildings they define over $\mathbf{Q}_{3}$ are the "same"; with suitable identifications of buildings and groups, $\Gamma^{b}=\Gamma_{\max }\left(e_{6}\right) \cap \Gamma_{\max }\left(b_{6}^{\prime}\right)$ has index 27 in $\Gamma_{\max }\left(e_{6}\right)$ and index 2 in $\Gamma_{\max }\left(b_{6}^{\prime}\right)$. Passage mod 2 maps $\Gamma_{\max }\left(e_{6}\right)$ onto $\operatorname{PGO}(5,3)$, and the preimages in $\Gamma_{\max }\left(e_{6}\right)$ of the 5 different classes of flag-transitive subgroups of $\operatorname{PGO}(5,3)$ (cf. $[\mathbf{S}]$ ) form the 5 desired conjugacy classes of groups $\Gamma$, exactly 3 of which have members in $\Gamma^{b}$. The 6 remaining conjugacy classes of chamber-transitive subgroups of $\Gamma_{\max }\left(b_{6}^{\prime}\right)$ not having members in $\Gamma_{\max }\left(e_{6}\right)$ consist of groups having index 1 or 2 in $\langle\Gamma, r\rangle$ for one of the chamber-transitive subgroups $\Gamma$ of $\Gamma^{b}$, where $r$ is the reflection $x_{6} \mapsto-x_{6}$.

Received by the editors May 21, 1986.

1980 Mathematics Subject Classification (1985 Revision). Primary 20G25, $20 \mathrm{H} 15$. 
(iii) Let $\Delta$ be the building of $G_{2}\left(\mathbf{Q}_{2}\right)$, and set $\Gamma=G_{2}(\mathbf{Z}[1 / 2])$ relative to the Z-form of $G_{2}$ corresponding to the standard Z-form of the usual Cayley division algebra over $\mathbf{Q}$.

(iv) Let $\Delta$ be the building of $\operatorname{PSL}\left(3, Q_{2}\right)$, and let $\Gamma$ be the subgroup $\operatorname{PSU}(h, \mathrm{Z}[1 / 2, \sqrt{-7}])<$ Aut $\Delta$, where $h$ is the $3 \times 3$ hermitian matrix

$$
\left(\begin{array}{lll}
1 & \mu & \mu \\
\bar{\mu} & 1 & \mu \\
\bar{\mu} & \bar{\mu} & 1
\end{array}\right)
$$

for $\mu$ satisfying $9 \mu^{2}+3 \mu+2=0$ and $\mu+\bar{\mu}=-1 / 3$.

(v) Let $\Delta$ be the building of $\operatorname{PSL}\left(3, \mathrm{~F}_{q}((t))\right), q=2$ or 8 . Consider the cyclic division algebra $D$ over $\mathbf{F}_{q}((t))$ generated by $\mathbf{F}_{q^{3}}((t))$ and an element $\sigma$ satisfying $\sigma^{3}=t+1, \sigma t=t \sigma$ and $\sigma x \sigma^{-1}=x^{3}$ for $x \in \mathbf{F}_{q^{3}}$. If $\Lambda$ is the order over $\mathbf{F}_{q}\left[t, t^{-1},(t+1)^{-1}\right]$ generated by $\mathbf{F}_{q^{3}}$ and $\sigma$, let $\Gamma$ be the group of $\mathbf{F}_{2}((t))$-algebra automorphisms of $D$ preserving $\Lambda$.

The examples in (i)-(v) were constructed in $[\mathbf{K 1}, 2, \mathrm{KaMW}, \mathrm{KMW1}, 2$, $\mathbf{M}, \mathbf{M u}, \mathbf{M W}, \mathbf{T 2}, \mathbf{W}]$.

THEOREM. Let $\Delta$ and $\Gamma$ be as in the first paragraph. Then $(\Gamma, \Delta)$ is isomorphic to one of the pairs enumerated in (i) $-(\mathrm{v})$.

Furthermore, if $\Delta$ is one of the buildings of (i)-(v), any chamber-transitive discrete subgroup of Aut $\Delta$ is conjugate to one of the above $\Gamma$ by an element of Aut $\Delta$; in other words, the normalizer of any such $\Gamma$ in $\Sigma$ Aut $\Delta$ satisfies $N_{\Sigma \text { Aut }}(\Gamma) \cdot$ Aut $\Delta=\Sigma$ Aut $\Delta$.

As discussed in [K3, T2], our theorem is closely related to results of Timmesfeld and Stroth prompted by the "revisionist" point of view in the classification of finite simple groups. However the methods of proof are very different.

2. Sketch of proof. In this section we will sketch the main part of the proof of the Theorem. However, exceptional situations that arise en route will not be pursued further: only the "generic" case will be considered in detail, and will be shown to lead to no examples of pairs $\Delta, \Gamma$. The small number of situations not dealt with here require intricate ad hoc arguments leading to the still smaller number of examples appearing in the Theorem.

Let $\mathcal{G}, \Delta, K$, and $\Gamma$ be as in the first paragraph of $\S 1$. Let $\mathbf{F}_{q}$ be the residue field of $K$, of characteristic $p \mid q$. Associated with $\Delta$ is an extended Dynkin diagram having $r \geq 3$ vertices. Let 1 and 2 denote vertices of $\Delta$ lying in a chamber $C$ and corresponding to a pair of end nodes of this diagram (or adjacent nodes, if the diagram is that of $\left.\tilde{A}_{r-1}\right)$. For $i=1,2$, the $\operatorname{star} \operatorname{Star}(i)$ is the finite spherical building of a semisimple group defined over $\mathbf{F}_{q}$. By excluding the $\operatorname{Sp}(4,2)$ and $G_{2}(2)$ buildings, we can assume that

(1) Aut $\operatorname{Star}(i)^{(\infty)}$ is the simple group associated with $\operatorname{Star}(i)$ for $i=1,2$

(where " $(\infty)$ " denotes the last term of the derived series).

Let $\Gamma_{i}$ be the stabilizer of $i$ in $\Gamma$, and let $K_{i}$ denote the kernel of the action of $\Gamma_{i}$ on $\operatorname{Star}(i)$, so that $\Gamma_{i} / K_{i}$ is a chamber-transitive subgroup of Aut $\operatorname{Star}(i)$. 
We will assume that

$$
\Gamma_{i} / K_{i} \geq \operatorname{Aut} \operatorname{Star}(i)^{(\infty)} \text { for } i=1,2,
$$

there being only a handful of possibilities for the building $\operatorname{Star}(i)$ for which (2) might fail, all of which were enumerated by Seitz [S].

Statement (2) allows one further useful reduction: $\Gamma_{i}^{(\infty)}$ is chambertransitive on $\operatorname{Star}(i)$, so that $\left\langle\Gamma_{1}^{(\infty)}, \Gamma_{2}^{(\infty)}\right\rangle$ is chamber-transitive on $\Delta$. Consequently, we may assume that

$$
\Gamma=\Gamma^{(\infty)}=\left\langle\Gamma_{1}^{(\infty)}, \Gamma_{2}^{(\infty)}\right\rangle
$$

Since $($ Aut $\Delta) / \mathcal{G}(K)^{\prime}$ is solvable, it follows that

$$
\Gamma<\mathcal{G}(K)^{\prime} \text {. }
$$

At this point, the proof splits into two very different major parts, according to whether char $K$ is 0 or $p$.

Case I. Char $K=0$. By (4), the finite group $\Gamma_{1}$ acts projectively on the smallest degree nontrivial projective module $V$ for $\mathcal{G}$ over $K$. By $(2), \Gamma_{1}^{(\infty)}$ is an extension of the simple group $\operatorname{Aut} \operatorname{Star}(1)^{(\infty)}$ by $K_{1} \cap \Gamma_{1}^{(\infty)}$. The results in [FT] imply that Aut $\operatorname{Star}(1)^{(\infty)}$ has a faithful complex irreducible projective representation of degree $\leq \operatorname{dim} V$. However, comparison of the pair $\left(\right.$ Aut $\operatorname{Star}(1)^{(\infty)}, \operatorname{dim} V$ ) with the table of minimal degree projective representations obtained in [LS] shows that our simple group Aut $\operatorname{Star}(1)^{(\infty)}$ belongs to a very small list (having $r \leq 5$ and $q \leq 3$ ). Since we are only considering the generic case of the Theorem, we can conclude that Case I does not occur. (N.B.-The possibilities left to be dealt with in detail can be limited further by replacing 1 by a suitably chosen vertex 2 and arguing similarly. Of course, in view of the statement of the Theorem, most actual examples fall under the nongeneric part of Case I.)

Before considering the case char $K \neq 0$, we note the following fact, valid in all characteristics.

The stabilizer $P_{x}$ of a vertex $x$ of $\Delta$ in a parabolic subgroup $P$ of $\mathcal{G}(K)$ does not act transitively on the chambers containing $x$.

Indeed, $P$ fixes a "point at infinity" $\xi$ of $\Delta$, therefore $P_{x}$ preserves the set of all chambers containing a segment $[x, y]$ of the half-line $[x, \xi]$, with $x \neq y$ (cf. [T4, Proposition 5; BrT, (2.5.6), (5.1.33), (4.2.5)] for the definitions of $\xi,[x, y]$, and $[x, \xi])$.

Case II. Char $K=p$. Let $S$ denote a Sylow $p$-subgroup of the stabilizer of the chamber $C$ in $\Gamma$, and consider first the case where $\mathcal{G}(K)=\operatorname{PGL}(V)$ for an $r$-dimensional vector space $V$ over a division algebra with center $K$. Label the vertices of $C$ by the integers $\bmod r$ so that the corresponding vertices of the Dynkin diagram of type $\tilde{A}_{r-1}$ are arranged cyclically in a natural order. Extending slightly the representation theory of $\operatorname{PSL}\left(n, p^{d}\right)\left(\cong\left(\Gamma_{i} / K_{i}\right)^{(\infty)}\right.$ for all $i$ and some $d$, by (2)) in characteristic $p$ so as to include representations over division algebras, one can show that $K_{i}=1$ for all $i$, that $S$ stabilizes a single 
point $X$ and a single hyperplane $Y$ of $V$, and that each $\Gamma_{i, i+1}=\Gamma_{i} \cap \Gamma_{i+1}$ (one of the "standard" maximal parabolic subgroups of $\Gamma_{i}$ ) stabilizes either $X$ or $Y$. Since $r \geq 3$, the stabilizer of $X$ or $Y$ contains two subgroups $\Gamma_{i, i+1}, \Gamma_{j, j+1}$ with $i \neq j, j+1$, so that $\Gamma_{i}=\left\langle\Gamma_{i, i+1}, \Gamma_{i, j, j+1}\right\rangle$ (as $\Gamma_{i, i+1}$ is maximal in $\Gamma_{i}$ ). This contradicts (5). Therefore the building $\Delta$ cannot be of type $\tilde{A}_{r-1}$.

In rough terms, the above argument says that the various groups $\Gamma_{i}$ "cannot cohabit" in PGL $(V)$. Similar (but often more intricate) arguments can be given for all $\mathcal{G}$, but the extended case analysis involved can be considerably reduced using an alternative approach sketched below, valid under the following hypothesis.

(*) For each vertex $i$ of $C$, a Sylow $p$-subgroup $U_{i}$ of $K_{i}$ is contained in a Borel subgroup of $\mathcal{G}$ defined over a separable extension of $K$.

By the Frattini argument, the normalizer $N_{i}$ of $U_{i}$ in $\Gamma_{i}$ acts chambertransitively on $\operatorname{Star}(i)$. In view of $(*)$ it follows that $U_{i}=1$, as otherwise $N_{i}$ would be contained in a proper parabolic subgroup of $\mathcal{G}(K)$ [BoT], contradicting (5). A glance through the tables in [T1] - or a simple general argument-shows that the equality of all $\left|\Gamma_{i}: K_{i}\right|_{p}$ can happen only if the diagram of $\Delta$ is of type $\tilde{A}_{r-1}$, namely, in the case treated above.

Fortunately, $(*)$ often holds [T3]. In particular, it is true whenever $\mathcal{G}$ is classical.

\section{REFERENCES}

[BoT] A. Borel and J. Tits, Éléments unipotents et sous-groupes paraboliques de groupes réductifs. I, Invent. Math. 12 (1971), 95-104.

[BrT] F. Bruhat and J. Tits, Groupes réductifs sur un corps local. I, Données radicielles valuées, Publ. Math. Inst. Hautes Études Sci. 41 (1972), 5-251.

[FT] W. Feit and J. Tits, Projective representations of minimum degree of group extensions, Canad. J. Math. 30 (1978), 1092-1102.

[K1] W. M. Kantor, Some exceptional 2-adic buildings, J. Algebra 92 (1985), 208-223.

[K2] _ Some locally finite flag-transitive buildings, European J. Combin. (to appear).

[K3] _ Generalized polygons, SCABs and GABS, Buildings and the Geometry of Diagrams: CIME Session, Como 1984 (L. A. Rosati, ed.), Lecture Notes in Math., vol. 1181, Springer-Verlag, 1986, pp. 79-158.

[KaMW] W. M. Kantor, T. Meixner, and M. Wester (in preparation).

[KMW1] P. Köhler, T. Meixner, and M. Wester, The 2-adic affine building of type $\tilde{A}_{2}$ and its finite projections, J. Combinatorial Theory (A) 38 (1985), 203-209.

[KMW2] _ The affine building of type $\tilde{A}_{2}$ over a local field of characteristic two, Arch. Math. 42 (1984), 400-407.

[LS] V. Landazuri and G. M. Seitz, On the minimal degrees of projective representations of the finite Chevalley groups, J. Algebra 32 (1974), 418-443. 1985.

[M] T. Meixner, Gruppen mit parabolischen Systemen, Habilitationsschrift, Giessen,

[MW] T. Meixner and M. Wester, Some locally finite buildings derived from Kantor's 2-adic groups, Comm. Algebra 14 (1986), 389-410.

[Mu] D. Mumford, An algebraic surface with $K$ ample, $\left(K^{2}\right)=9, p_{g}=q=0$, Amer. J. Math 101 (1979), 233-244. 
[S] G. M. Seitz, Flag-transitive subgroups of Chevalley groups, Ann. of Math. (2) 97 (1973), 27-56; correction (unpublished).

[T1] J. Tits, Reductive groups over local fields. Proc. Sympos. Pure Math., vol. 33, part I, Amer. Math. Soc., Providence, R.I., 1979, pp. $29-69$.

[T2] _ Buildings and group amalgamations, Proc. St. Andrews Group Theory Conf., 1985 (C. Campbell and E. Robertson, eds.), Cambridge Univ. Press (to appear).

[T3] _ Éléments unipotents et sous-groupes paraboliques de groupes réductifs. II, Proc. Conf. Algebraic Groups, Utrecht, 1986 (to appear).

[T4] , Immeubles de type affine, Buildings and the Geometry of diagrams: CIME Session, Como 1984 (L. A. Rosati, ed.), Lecture Notes in Math., vol. 1181, Springer-Verlag, 1986, pp. 159-190.

[W] M. Wester, Endliche fahnentransitive Tits-Geometrien und ihre universellen Überlagerungen, Mitt. Math. Sem. Giessen 170 (1985), 1-143.

DePARTMENT OF MATHEMATICS, UNIVERSity OF OREgon, EUGENE, OREgoN 97403

DEPARTMENT OF MATHEMATICS, COLORADO STATE UNIVERSity, ForT COLlins, COLORADO 80523

Collège de France, 75231 Paris Cedex 05, France 
